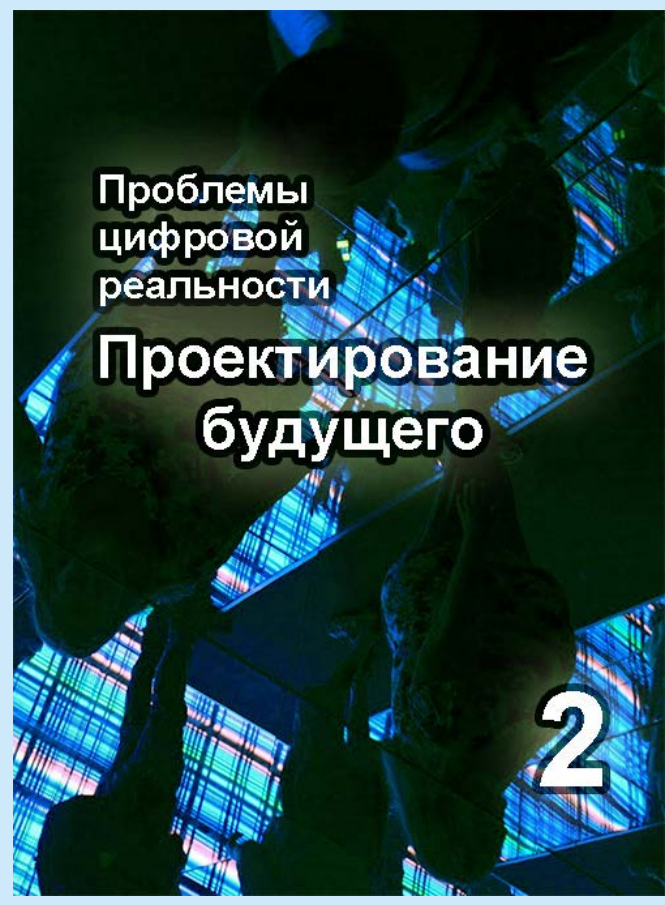

\title{
Н.С. Ильюшенко
}

Digital learning: Перспективы и риски цифрового поворота в образовании

Рекомендуемая форма библиографической ссылки

Ильюшенко H.C. Digital learning: Перспективы и риски цифрового поворота в образовании // Проектирование будущего. Проблемы цифровой реальности: труды 2-й Международной конференции (7-8 февраля 2019 г., Москва). - М.: ИПМ им. М.В.Келдыша, 2019. - С. 215-225.

— URL: https://keldysh.ru/future/2019/20.pdf doi:10.20948/future-2019-20 


\title{
Digital learning: Перспективы и риски цифрового поворота в образовании
}

\author{
Н.С. Ильюшенко \\ Государственное научное учреждение «Институт философии \\ Национальной академии наук Беларуси»
}

\begin{abstract}
Аннотация. Статья посвящена экспликации и оценке перспектив и рисков цифрового поворота в образования. Конкретизировано понятие цифрового поворота в обучении, произведен сравнительный анализ широко используемых в теоретической литературе категорий «цифровое обучение» (digital learning), «электронное обучение» (e-learning), «онлайнобучение» (online-learning), «виртуальное обучение» (virtual learning), «дистанционное обучение» (distance learning) и др. Представлена дефиниция цифрового обучения. Рассмотрена эволюция форм и идей, положенных в основу практик digital learning. Описаны ведущие тенденции цифрового обучения в 2017-2018 гг. Отмечены наиболее значимые направления развития цифрового обучения с учетом целей повышения качества образования в Республике Беларусь. Сформулированы предложения по минимизации негативного воздействия цифровизации на образовательный процесс.
\end{abstract}

Ключевые слова: цифровизация, цифровой поворот, цифровое обучение, образовательный процесс

\section{Digital learning: Prospects and risks of digital turn in education}

\author{
N.S. Ilyushenka \\ State Scientific Institution «Institute of Philosophy \\ of the National Academy of Sciences of Belarus»
}

\begin{abstract}
The prospects and risks of the digital turn in education are analyzed in the article. The concept of digital turn in education is specified. A comparative analysis of the categories "digital learning", "e-learning", "online learning", "virtual learning", "distance learning" widely used in theoretical literature is made. The definition of digital learning is presented. The evolution of the forms and ideas constituting the basis of digital learning is considered. The leading trends of 2017-2018 in digital learning are described. The most significant directions of development of digital education are noted, taking into
\end{abstract}


account the goals of improving the quality of education in the Republic of Belarus. The proposals to minimize the negative impact of digitalization on the educational process are proposed.

Keywords: digitalization, digital turn, digital learning, educational process

Актуальность изучения перспектив и рисков перехода к цифровому обучению (digital learning) определяется рядом факторов.

В первую очередь, она связана с вызовами современного глобального мира, стремительностью происходящих сегодня технологических и социальных трансформаций, требующих применения наиболее быстрых и экономически выгодных способов генерации и передачи знаний. Цифровое обучение как инструмент перестройки и обновления системы образования соответствует этим целям. Сегодня оно все чаще признается в качестве средства, способного обеспечить достижение целей устойчивого развития (ЦУР), инструмента поддержания креативного и инновационного потенциала жителей планеты на должном уровне. В частности, в Декларации Циндао (2015) подчеркивается, что информационнокоммуникативные технологии (ИКТ) являются ключом к достижению ЦУР 4 «Образование» [1]. Исследования Global e-Sustainability Initiative (GeSI) (2016) подтвердили, что цифровые решения способны оказать существенное положительное влияние на каждую из 17 целей, в том числе на трансформацию образовательных возможностей людей $[2$, p.13]. В подтверждение тезиса о востребованности развития цифрового образования приведем также данные исследования, организованного компанией «Делойта» в области международных тенденций развития человеческого капитала за 2017 г. Опрос более 10 тысяч руководителей компаний и экспертов из 140 стран, показал, что $83 \%$ компаний считают необходимым введение цифрового обучения сотрудников и менеджеров для улучшения работы компаний, а $54 \%$ - считают такую меру срочной, что на $11 \%$ больше по сравнению с данными 2016 г. [3].

Не менее значимым аргументом в пользу анализа перспектив и рисков имплементации цифровых технологий в образовательную практику выступают задачи самого образования, которое сегодня должно не только идти в ногу со временем, но и во многом опережать его. Нынешним школьникам и студентам предстоит жить и работать фактически в будущем, что означает, что их подготовка должна соответствовать потребностям трудового рынка не сегодняшнего, а завтрашнего дня. Дети и молодежь должны быть готовы к работе в мире, где все больше сфер деятельности и труда затронуто цифровизацией. Это значит, что образовательный процесс должен быть перестроен с учетом стоящей перед ним необходимости реформирования своих сущностных основ, включая приведение методик и форм обучения в соответствие с требованиями наступающей цифровой эпохи. Показательным здесь является 


\section{5. Образование в цฺифровой реальности}

предложенное Марком Пренски разделение людей на «цифровых иммигрантов» и «цифровых коренных жителей (аборигенов)» [4]. По определению М. Пренски, цифровой иммигрант - это взрослый человек, который свободно пользуется современными цифровыми технологиями, в то время как коренное население - это молодые люди, которые выросли с интернетом и не помнят свою жизнь без него. По мнению автора, эти люди живут в мире, в который естественным образом встроена мобильность, интерактивность и различные цифровые каналы коммуникации. Но образовательные программы до сих пор создают люди, во многом далекие от этого пласта реальности. Представляется важным произвести интеграцию этих двух областей жизни (цифровой и нецифровой) таким образом, чтобы люди поколения Z или Y, как их по-разному называют, воспринимали обучение как естественное, а не ограничивающее их продолжение жизни. Однако сделать это важно не путем отрицания всего накопленного ранее педагогического опыта. Должна быть установлена мера, произведен анализ того, где проходит граница, после пересечения которой перевод образования «в цифру» не повышает, а, напротив, снижает качество подготовки обучающихся.

На значимость изучения темы указывает и отсутствие консенсуса о роли и задачах цифрового образования при все возрастающем интересе к нему со стороны государственных и коммерческих организаций. Сегодня как никогда слышны голоса тех, кто ратует за всепоглощающую цифровизацию образования. Вместе с тем обращают на себя внимание набирающие силу алармистские настроения, предупреждающие об опасностях, которые принес с собой цифровой поворот. Так, заявляет о себе необходимость оценки не только плюсов, но и возможных негативных последствий цифровых трансформаций, реализуемых в сфере образования. Особенно важно дать ответ на вопрос о возможных угрозах перед внедрением цифровых технологий в практику начальной школы, где объектом воздействия выступают дети с еще не полностью сформированными учебными навыками.

Прежде чем описать ключевые черты цифрового поворота в образовании, важно понять, что вообще представляет собой это явление. Отметим, что оно не имеет универсальной трактовки. Одними исследователями цифровой поворот определяется как процесс развития цифровых технологий, а другими - как теоретический инструмент, позволяющий осмыслить то, как цифровизация в целом влияет на общество, трансформируя все его сферы $[5$, p.16]. При этом в последние годы закрепилась традиция выделять два цифровых поворота. Первый непосредственное привнесение технических инноваций в ту или иную сферу жизни - существенно не повлиял на сами практики, переведя процесс создания продукта из ручного режима, например, в машинный. Второй проявился в трансформации самих сфер жизнедеятельности, 
которые произошли под влиянием цифровизации. Их следствием выступило появление новых практик взаимодействия и коммуникации, способов мышления, организации работы и повседневной деятельности на сущностном уровне.

Описывая специфику цифрового поворота в сфере образования, следует отметить, что он проявился в создании новой инфраструктуры, распространении новых (цифровых) технологий и устройств обучения, а также в их повсеместном внедрении в учебный процесс; трансформации подходов и техник работы педагогов с обучающимися; изменении требований к самому процессу обучения и ожиданий от него.

Говоря о сущности феномена цифрового обучения как результате цифрового поворота в образовании, важно указать на то, что отсутствие общего определения обусловливает различия в интерпретации digital learning. Нередко цифровое обучение отождествляется с такими близкими понятиями, как: e-learning (электронное обучение), online-learning (онлайн обучение), virtual learning (виртуальное обучение), distance learning (дистанционное обучение), the Smart Teaching Technique и др. Одновременно обнаруживает себя тенденция к противопоставлению цифрового обучения данным формам образовательной деятельности. Приведем несколько примеров. В частности, электронное обучение предлагается относить к миру образования, в то время как цифровое обучение - к миру бизнеса [6]. Также уже сложилась традиция рассматривать digital learning как стадию развития e-learning [7]. В данной статье мы будем определять цифровое образование широко, не отождествляя его при этом с представленными выше понятиями. Digital learning - стадия развития образовательных и учебных практик, предполагающих применение разнообразных стратегий обучения, базирующихся на использовании цифровых технологий и служащих повышению эффективности образовательного процесса. Цифровое обучение, таким образом, включает в себя концепции онлайн-обучения, электронного обучения, дистанционного обучения и др. (оно также включает в себя то, что может быть названо «цифровым обучением в автономном режиме», то есть обучение с использованием локального программного обеспечения и цифровых камер, не предполагающих непосредственный выход в Интернет).

В качестве своих инструментов digital learning включает средства мультимедиа, интерактивные учебные курсы, онлайн-курсы, онлайн базы, обучающие среды, системы управления образованием (LMS), системы смешанного или гибридного обучения, использование цифрового контента и Интернет-ресурсов, использование технологий дополненной и виртуальной реальностей, анализа больших данных, искусственного интеллекта, машинного обучения, достижений робототехники [8]. Многообразие средств, которые могут быть использованы в ходе 


\section{5. Образование в изифровой реальности}

цифрового обучения, позволяет заключить, что цифровая стратегия обучения характеризуется широкой вариативностью ее реализации.

Рассматривая специфику digital learning, нельзя оставить в стороне вопрос эволюции цифрового образования. Представляется возможным выделить четыре стадии развития:

Первая стадия (конец 1980-х гг.) - этап компьютерного обучения или обучения с использованием компьютерной техники - здесь основу образовательного процесса составляло выполнение обучающимися отдельных заданий и упражнений для формирования и тренировки тех или иных навыков.

Вторая стадия (со 2-й половины 1990-х гг.) - этап электронного обучения (e-learning) или обучения с использованием электронных средств и технологий - предполагала применение CD-ROM, аудио/видеокассет, интерактивного телевидения, спутникового вещания, интранета, интернета; эволюция на этой стадии происходила в соответствии с популяризацией следующих форм обучения:

1) курсов на носителях CD-ROM;

2) дистанционных занятий с преподавателями;

3) курсов, предполагающих использование специальных интерактивных программ (в том числе, электронных учебников);

4) онлайн-курсов (включая появление феномена онлайнуниверситетов), базирующихся на различных образовательных платформах и системах управления обучением, виртуальных образовательных платформах;

5) мобильных приложений, видеоресурсов, социальных сетей, платформ-агрегаторов онлайн курсов (например, Coursera и $\mathrm{EdX}$ и др.).

Третья стадия (с 2015 г.) - этап собственно digital learning - обучение не «с помощью», но «через / посредством» использования цифровых технологий. Она объединяет весь накопленный на предшествующих стадиях опыт.

Четвертая стадия (прогно3) (с 2020 г.) - intelligent learning или интеллектуальное обучение - предполагает переход на индивидуализированное, персонализированное обучение, реализуемое с участием искусственного интеллекта, а также с учетом когнитивных особенностей запоминания информации человеком: например, интервальное обучение и др. [7].

Эволюция идей, положенных в основу цифрового образования, и технологических достижений, позволяющих осуществить их реализацию, может быть представлена следующим образом.

1) переход от принципа «self-study» к принципу «everyone, everywhere, all the time» (обучение по требованию, непрерывный процесс саморазвития вне зависимости от места пребывания адресата); 
2) переход от практики передачи информации и инструкций (дизайн обучения) к получению реального опыта (дизайн опыта) (от теории - к практике);

3) переход от идеи «каталога ресурсов», «места хранения данных»к «потоку изменений»;

4) переход от традиционных LMS-платформ к мобильным и «невидимым» версиям (разработанным на основе социальных сетей и цифровых медиа).

При постановке вопроса о направленности эволюции цифрового образования можно отметить проникновение технологий сначала из сферы обучения взрослых в практику вузов, потом, во все большей степени, - в школы (от старших, до начальных классов).

Оценивая тенденции цифрового образования в 2017-18 гг., представляется возможным выделить наиболее значимые из них:

- внедрение в образовательную практику социальных сетей и социальных медиа (около 40\% школ допускают использование социальных сетей только в учебных целях, почти $20 \%$ разрешают открытое использование социальных сетей в школе, что свидетельствует о том, что все большее количество учреждений осознают, что пришло время встретить обучающихся там, где они «актуально присутствуют»);

- применение мобильных устройств в качестве средств цифрового обучения;

- углубление кооперативной работы педагогов, ученых, программистов, медиков и представителей других профессий для разработки более эффективных образовательных систем;

- введение в штат учебных организаций новых сотрудников (учебные / образовательные технологи; академические координаторы и др.), задача которых состоит в планировании, организации и контроле за реализацией проектов цифрового образования в учреждениях образования.

Анализируя положительные следствия цифрового поворота в образовании, укажем, прежде всего, на возможности дистанционного (удаленного) и гибкого обучения, что важно, в первую очередь, для больших фирм, где сотрудников сложно собрать всех в одном месте и в одно время. Также такое обучение существенно повышает доступность (инклюзивность) образования, позволяя получить образовательные услуги тем, кто по различным причинам не может физически присутствовать в учебном классе или аудитории. Возможности выбора времени обучения позволяют получить знания в удобный для этого момент. Не менее значимым положительным моментом является расширение аудитории получателей образовательных услуг - так качественные знания может получить большее количество людей при сравнении, например, с возможностями одного педагога в одной аудитории. Цифровое обучение также служит повышению интерактивности образовательного процесса, 


\section{5. Образование в изифровой реальности}

что положительно влияет на его эффективность, поскольку доказано, что интерактивные формы обучения приводят к более высоким результатам, чем использование традиционных методик. Это особенно важно при обучении взрослых, которым бывает сложно усвоить новую информацию. Digital learning признается в качестве средства индивидуализации образования, позволяя реализовать персонифицированную стратегию обучения, откорректировать темп освоения материала, отобрать наиболее подходящие для этого методики и техники. Оно также открывает возможности тиражирования и распространения качественного цифрового контента - получаемая информация больше не зависит от настроения, здоровья педагога. Как правило, контент разрабатывается таким образом, чтобы его было легко воспринимать - читать и понимать, поскольку подача информации сопровождается видео и изображениями для ее лучшего усвоения. Digital learning позволяет осуществить датификацию результатов - возможность быстрого анализа достижений и/или сложностей как отдельного ученика, так и всей группы. Оно помогает отслеживать успеваемость, обеспечивать прозрачность учебного процесса для всех заинтересованных участников. При этом цифровое обучение позволяет экономить денежные средства, инфраструктурные и человеческие ресурсы (например, онлайн-курсы и другие цифровые инструменты позволяют менеджерам в разных местах обучаться одновременно, экономя компании время и командировочные расходы).

Оценивая, кто выигрывает от цифрового поворота в образовании, исследователи называют всех тех, кто напрямую или косвенно заинтересован в успешности образовательного процесса - обучающихся, учителей, родителей, менеджеров, руководителей организаций и компаний, государства в целом. Так, например, исследование К-12 «Глобальное состояние цифрового обучения», проведенное с участием 9279 учителей и администраторов из 89 разных стран, выявило, что в подавляющем большинстве респонденты согласны с утверждением, что цифровое обучение положительно влияет на обучающихся и преподавателей [9].

При этом нельзя обойти стороной проблему злоупотребления цифровыми средствами в процессе обучения. Существуют обоснованные сомнения в том, что полный перевод обучения «в цифру» будет благоприятен, особенно для детей младшего школьного возраста. Необходим поиск баланса между цифровыми и традиционными технологиями, основанными на реальных коммуникативных практиках (общение «лицом к лицу) и взаимодействии участников образовательного процесса. Ведь сущность концепции цифрового обучения состоит не в том, чтобы просто предоставить студентам доступ к технологиям или заменить традиционные методы на передовые, технологически емкие, но произвести 
вдумчивую интеграцию технологий в учебный процесс таким образом, чтобы действительно улучшить его результаты.

Следует отметить, что, несмотря на признанные положительные результаты использования инструментов digital learning, специфику цифрового поворота во многом определяют не столько возможности цифрового образования, сколько те новые риски, которые принесла с собой цифровизация учебной деятельности. И хотя, как указывалось выше, имеется много свидетельств эффективности цифрового обучения, ряд исследователей приводят доводы в защиту тезиса о серьезных проблемах, сопутствующих применению концепции digital learning в качестве всеобщего принципа организации образования. В частности, $34 \%$ респондентов, участвующих в уже отмеченном опросе К-12 «Глобальное состояние цифрового обучения», хотя и считают интеграцию новых инструментов edtech главным приоритетом, тем не менее, страдают от последствий «технологического раздувания» или слишком быстрого и масштабного включения технологий в учебный процесс [9].

Таким образом, в качестве ключевых рисков могут быть отмечены:

1) Использование большого количества инструментов для решения педагогических задач создает дополнительную учебную и рабочую нагрузку для обучающихся и учителей, поскольку заставляет их тратить время на поиск, установку, освоение нового программного и технического обеспечения, что обычно не предусмотрено образовательными программами. Интересно отметить, что «раздувание технологий», в котором приходится манипулировать слишком большим количеством инструментов, увеличилось почти на 8\% в 2018 г., а место доступа к технологиям стало самой большой проблемой для учителей. Это же обстоятельство подрывает требование сохранения единства образовательного процесса - разные учителя могут пользоваться разными средствами, что создает препятствия на пути достижения единообразия педагогических практик, может затруднять адаптацию при переходе из одного учебного заведения в другое.

2) Большие временные затраты на создание цифрового образовательного продукта и его быстрое устаревание приводит к потере качества контента. При этом, если мы говорим о педагогах вузов и школ, то цифровизация образования не ликвидирует образовательные программы, утвержденные на государственном уровне, а также не снимает с педагога обязанность по проведению занятий в традиционной форме (это может создавать двойную нагрузку на педагога: необходимость готовить материалы к занятиям в цифровой и «традиционной» форме, дублировать отчетность и т.д.).

3) Цифровизация также может приводить к игнорированию ряда передовых педагогических идей, которые с трудом подлежат оцифровыванию. Здесь же можно отметить снижение качества 


\section{5. Образование в цฺифровой реальности}

сформированности умений, которые развиваются только в коммуникации «лицом к лицу» - социальных навыков, навыков межличностного общения; а также утрату способностей воспринимать большие тексты, писать и др.

4) Цифровизация образования ставит проблемы информационной безопасности, защиты авторских прав и защиты персональных и учебных данных обучающихся.

5) Цифровизация также нередко называется в качестве основы развития новых форм дискриминации педагогов, которым в силу возраста сложно адаптироваться к трансформирующейся цифровой реальности, а также обучающихся, которые не имеют необходимых средств обучения (качественных технических устройств, дорогостоящего программного обеспечения и др.).

6) В качестве психологических рисков отмечается вероятность развития зависимости от гаджетов.

Таким образом, цифровизация не способна решить многие проблемы неэффективности существующих образовательных институтов.

Говоря о возможностях снижения рисков цифровизации в сфере образования, представляется возможным выделить несколько перспективных направлений.

1) Целенаправленное развитие цифровой педагогики как самостоятельного направления педагогической науки, учитывающей влияние «цифровой реальности» на поведение человека, нормы социальных отношений, практики восприятия и усвоения информации. Только на этой основе можно будет осуществить рефлексивное внедрение цифровых технологий в образовательный процесс. В этой связи необходимо отметить важность решения целого ряда как теоретических, так и практических задач. В первую очередь, переосмыслить роли и функции учителя и обучающихся, образовательных учреждений и учебных материалов. Особого решения потребует задача разработки и внедрения курсов, ориентированных на обучение самих педагогов. Эти курсы должны учить, как разрабатывать электронные образовательные курсы, эффективно использовать их в своей профессиональной деятельности для достижения наиболее высоких результатов, учитывая при этом персональные запросы обучающихся.

2) На организационном уровне должны быть созданы условия для взаимодействия всех участников процесса разработки и внедрения цифровых образовательных средств (субъекты, ответственные за принятие решений на государственном уровне; учебные заведения; разработчики образовательных программ; органы, обеспечивающие качество сертификации и др.). Учитывая встревоженность общественности последствиями чрезмерной увлеченности цифровыми технологиями, представляется важным провести лонгитюдные исследования о влиянии 
гаджетов на здоровье и сознание пользователей (особенно детей) - на сегодняшний день очевидна их нехватка. До получения результатов рекомендуется уравновешивание цифровых форм взаимодействий и обучения нецифровыми, взаимодействием «лицом к лицу» в живой межличностной коммуникации (примером программы, направленной на развитие таких способностей, может считаться образовательная программа для детей и подростков «Зеленое Солнце», разработанная коллективном авторов Института философии НАН Беларуси).

3) Ключевым моментом в дальнейшем продвижении практик цифрового обучения должно стать обеспечение информационной безопасности, усовершенствования правого регулирования данной сферы, а также стимулирование развития цифрового правосознания в области реализации новых образовательных практик (внедрение курсов медиа компетентности и др.).

В заключение отметим несколько значимых шагов в направлении развития цифрового образования в Республике Беларусь. В первую очередь, следует отметить курс на построение IT-страны, разработку концепции цифровой трансформации образования, внедрение инноваций в практику обучения в школах и высших учебных заведениях. В Беларуси установлена традиция проведения ежегодных научных мероприятий, симпозиумов, выставок, посвященных цифровому образованию. В качестве наиболее значимого примера таких мероприятий можно назвать Международную специализированную научно-техническую выставкуфорум «Информационные технологии в образовании». В 2018 г. в выставке приняли участие более 70 спикеров и почти 2 тысячи участников, она объединила самые современные разработки, которые внедряются в учебный процесс. Также в стране набирает популярность традиция празднования Digital Learning Day, установленного в 2012 г. для привлечения внимания к эффективному использованию современных средств обучения для улучшения образования в государственных школах. В марте 2016 г. под эгидой ЮНЕСКО впервые была проведена Неделя обучения с помощью цифровых устройств.

Подводя итоги, следует сказать, что цифровизация - это глобальный процесс, поэтому невозможно заниматься развитием цифрового образования на уровне отдельно взятой школы или компании - необходим гораздо более широкий подход. В реализации задач цифровизации образования должны принимать участие ученые, специалисты ИТкомпаний, разработчики программного обеспечения, педагоги и, конечно, сама общественность.

\section{Литература}

1. Qingdao Declaration (2015): Seize Digital Opportunities, Lead Education Transformation / UNESCO Institute for Information Technologies in 
Education.

URL:

https://millenniumedu.files.wordpress.com/2018/11/unesco-qingdaodeclaration-2015.pdf. $-54 \mathrm{p}$.

2. \#Systemtransformation: How Digital Solutions Will Drive Progress Towards The Sustainable Development Goals. - URL: http://systemtransformationsdg.gesi.org/160608_GeSI_SystemTransformation.pdf. - 40 p.

3. Deloitte's fifth annual Global Human Capital Trends report. Rewriting the rules for the digital age (2017). - URL: https://www2.deloitte.com/content/dam/Deloitte/global/Documents/Huma nCapital/hc-2017-global-human-capital-trends-gx.pdf. - 140 p.

4. Prensky, M. Digital Natives, Digital Imigrants, Part 1. // On the Horizon. - 2001. - Vol. 9. - №. 5. - № 5. - P. 3-6.

5. The Digital Turn in Higher Education: International Perspectives on Learning and Teaching in a Changing World / Ed. by Kergel, D., Heidkamp, B., Telléus, P.K., Rachwal, T., Nowakowski, S. - VS Verlag für Sozialwissenschaften, 2018. - $239 \mathrm{p}$.

6. Corporate learning, digital learning or elearning? / DOKEOS. - URL: https://www.dokeos.com/corporate-learning-digital-learning-orelearning/.

7. Bersin J. The Disruption of Digital Learning: Ten Things We Have Learned - URL: https://joshbersin.com/2017/03/the-disruption-of-digitallearning-ten-things-we-have-learned/.

8. Every Student Succeeds Act: S.1177 - 114th Congress (2015-2016) // Public Law No: 114-95 (12/10/2015) 114th Congress Public Law 95. URL: https://www.congress.gov/bill/114th-congress/senatebill/1177/text\#toc-H40B9ACB8EFC8452FB4513086BEC0BB19.

9. Davis L. Digital Learning: What to Know in 2019 / Evolving Ed. January 25, 2019. - URL: https://www.schoology.com/blog/digital-learning-whatknow-2019. 